\title{
GENERALIZED CUT AND METRIC POLYTOPES OF GRAPHS AND SIMPLICIAL COMPLEXES
}

\author{
MICHEL DEZA AND MATHIEU DUTOUR SIKIRIĆ
}

\begin{abstract}
Given a graph $G$ one can define the cut polytope $\operatorname{CUTP}(G)$ and the metric polytope $\operatorname{METP}(G)$ of this graph and those polytopes encode in a nice way the metric on the graph. According to Seymour's theorem, $\operatorname{CUTP}(G)=\operatorname{MeTP}(G)$ if and only if $K_{5}$ is not a minor of $G$.

We consider possibly extensions of this framework:

(1) We compute the $\operatorname{CUTP}(G)$ and $\operatorname{MeTP}(G)$ for many graphs.

(2) We define the oriented cut polytope $\operatorname{WOMCUTP}(G)$ and oriented multicut polytope $\operatorname{OMCUTP}(G)$ as well as their oriented metric version $\operatorname{QMETP}(G)$ and $\operatorname{WQMeTP}(G)$.

(3) We define an $n$-dimensional generalization of metric on simplicial complexes.
\end{abstract}

\section{INTRODUCTION}

The cut polytope [23] is a natural polytope arising in the study of the maximum cut problem 10. The cut polytope on the complete graph $K_{n}$ has seen much study (see [23]) but the cut polytope on a graph was much less studied [20, 4, 2, Moreover, generalizations of the cut polytope on graphs seems not to have been considered.

Given a graph $G=(V, E)$, for a vertex subset $S \subseteq V=\{1, \ldots, n\}$, the cut semimetric $\delta_{S}(G)$ is a vector (actually, a symmetric $\{0,1\}$-matrix) defined as

$$
\delta_{S}(x, y)= \begin{cases}1 & \text { if }(x y) \in E \text { and }|S \cap\{x, y\}|=1, \\ 0 & \text { otherwise }\end{cases}
$$

A cut polytope $\operatorname{CUTP}(G)$, respectively cut cone $\operatorname{CUT}(G)$, are defined as the convex hull of all such semimetrics, respectively positive span of all non-zero ones among them. The dimension of $\operatorname{CUTP}(G)$ and $\operatorname{CUT}(G)$ is equal to the number of edges of $G$.

The metric cone $\operatorname{MET}\left(K_{n}\right)$ is the set of all semimetrics on $n$ points, i.e., the functions $d:\{1, \ldots, n\}^{2} \rightarrow \mathbb{R}_{\geq 0}$ (actually, symmetric matrices over $\mathbb{R}_{\geq 0}$ having only zeroes on the diagonal), which satisfy all $3\left(\begin{array}{l}n \\ 3\end{array}\right)$ triangle inequalities $d(i, j)+$ $d(i, k)-d(j, k) \geq 0$. The bounding of $\operatorname{MET}\left(K_{n}\right)$ by $\left(\begin{array}{l}n \\ 3\end{array}\right)$ perimeter inequalities $d(i, j)+d(i, k)+d(j, k) \leq 2$ produces the metric polytope $\operatorname{METP}\left(K_{n}\right)$.

For a graph $G=(V, E)$ of the order $|V|=n$, let $\operatorname{MET}(G)$ and $\operatorname{METP}(G)$ denote the projections of $\operatorname{MET}\left(K_{n}\right)$ and $\operatorname{METP}\left(K_{n}\right)$, respectively, on the subspace $\mathbb{R}^{|E|}$ indexed by the edge set of $G$. Clearly, $\operatorname{CUT}(G)$ and $\operatorname{CUTP}(G)$ are projections of, respectively, $\operatorname{CUT}\left(K_{n}\right)$ and $\operatorname{CUTP}\left(K_{n}\right)$ on $\mathbb{R}^{E}$. It holds

$$
\operatorname{CUT}(G) \subseteq \operatorname{MET}(G) \text { and } \operatorname{CUTP}(G) \subseteq \operatorname{MeTP}(G) .
$$

Key words and phrases. max-cut problem, cut polytope, metrics, graphs, cycles, quasi-metrics, hemimetrics. 
In Section 2 we consider the structure of those polytopes and give the description of the facets for many graphs (see Tables 1 and 2). The data file of the groups and orbits of facets of considered polytopes is available from [24].

The construction of cuts and metrics can be generalized to metrics which are not necessarily symmetric are considered in Section [3 (see also [19, 16]). The triangle inequality becomes $d(i, j) \leq d(i, k)+d(k, j)$ and the perimeter inequality becomes $d(i, j)+d(j, k)+d(k, i) \leq 2$ for $1 \leq i, j, k \leq n$. We also need the inequalities $0 \leq d(i, j) \leq 1$. The quasi metric polytope $\operatorname{QMETP}\left(K_{n}\right)$ is defined by the above inequalities and the quasi metric cone $\operatorname{QMET}\left(K_{n}\right)$ is defined by the inequalities passing by zero. The quasi metric cone $\operatorname{QMET}(G)$ and polytope $\operatorname{QMETP}(G)$ are defined as projection of above two cone and polytopes. In Theorem 3 we give an inequality description of those projections.

Given an ordered partition $\left(S_{1}, \ldots, S_{r}\right)$ of $\{1, \ldots, n\}$ we defined an oriented multicut as:

$$
\delta^{\prime}\left(S_{1}, \ldots, S_{r}\right)_{x, y}= \begin{cases}1 & \text { if } x \in S_{i}, y \in S_{j} \text { and } i<j \\ 0 & \text { otherwise }\end{cases}
$$

The convex cone of the oriented multicut is the oriented multicut cone OMCUT $\left(K_{n}\right)$. The convex polytope can also be defined but there are vertices besides the oriented multicuts. A smaller dimensional cone $\operatorname{WQMET}(G)$ and polytope $\operatorname{WQMETP}(G)$ can be defined by adding the cycle equality

$$
d(i, j)+d(j, k)+d(k, i)=d(j, i)+d(k, j)+d(i, k)
$$

to the cone $\mathrm{QMET}(G)$ and polytope $\operatorname{QMETP}(G)$. A multicut satisfies the cycle equality if and only if $r=2$. We note the corresponding cone $\operatorname{WOMCUT}(G)$ and WOMCUTP $(G)$. In Section 3 we consider those cones and polytopes and their facet description.

The notion of metrics can be generalized to more than 2 points and we obtain the hemimetrics. Those were considered in [15, 14, 17, 21]. Only the notion of cones makes sense in that context. The definition of the above papers extends the triangle inequality in a direct way: It becomes a simplex inequality with the area of one side being bounded by the sums of area of the other sides. In 12 we argued that this definition was actually inadequate since it prevented right definition of hemimetric for simplicial complex. In Section 4 we give full details on what we argue is the right definition of hemimetric cone.

There is much more to be done in the fields of metric cones on graphs and simplicial complexes. Besides further studies of the existing cones and the ones defined in this paper, two other cases could be interesting. One is to extend the notion of hypermetrics cone $\operatorname{HYP}\left(K_{n}\right)$ to graphs; several approaches were considered in [18], for example projecting only on the relevant coordinates, but no general results were proved.

Another generalization that could be considered is the diversities considered in [7, 8. Diversity cone $D I V_{n}$ is the set of all diversities on $n$ points, i.e., the functions $f:\{A: A \subseteq\{1, \ldots, n\}\} \rightarrow \mathbb{R}_{\geq 0}$ satisfying $f(A)=0$ if $|A| \leq 1$ and

$$
f(A \cup B)+f(B \cup C) \geq f(A \cup C) \text { if } B \neq \emptyset .
$$

The induced diversity metric $d(i, j)$ is $f(\{i, j\})$. 
Cut diversity cone $C D I V_{n}$ is the positive span of all cut diversities $\delta(A)$, where $A \subseteq\{1, \ldots, n\}$, which are defined, for any $S \subseteq\{1, \ldots, n\}$, by

$$
\delta_{S}(A)= \begin{cases}1 & \text { if } A \cap S \neq \emptyset \text { and } A \backslash S \neq \emptyset \\ 0 & \text { otherwise. }\end{cases}
$$

$C D I V_{n}$ is the set of all diversities from $D I V_{n}$, which are isometrically embeddable into an $l_{1}$-diversity, i.e., one, defined on $\mathbb{R}^{m}$ with $m \leq\left(\begin{array}{c}n \\ \left\lfloor\frac{n}{2}\right\rfloor\end{array}\right)$ by

$$
f_{m 1}(A)=\sum_{i=1}^{m} \max _{a, b \in A}\left\{\left|a_{i}-b_{i}\right|\right\} .
$$

These two cones are extensions of the $\operatorname{MET}\left(K_{n}\right)$ and $\operatorname{CUT}\left(K_{n}\right)$ on a complete hypergraphs and it would be nice to have a nice definition on any hypergraph.

\section{Structure of CUT POLYTOPES OF GRAPHS}

The cut metric $\delta_{S}$ defined at Equation (11) satisfies the relation $\delta_{\{1, \ldots, n\}-S}=\delta_{S}$. The cut polytope $\operatorname{CUTP}\left(K_{n}\right)$ is defined as the convex hull of the metrics $\delta_{S}$ and thus has $2^{n-1}$ vertices.

For a given subset $S$ of $\{1, \ldots, n\}$ we can define the switching operation $F_{S}$ by

$$
F_{S}(d)(i, j)=\left\{\begin{array}{cl}
1-d(i, j) & \text { if }|S \cap\{i, j\}|=1 \\
d(i, j) & \text { otherwise. }
\end{array}\right.
$$

The operation on cuts is $F_{S}\left(\delta_{T}\right)=\delta_{S \Delta T}$ with $\Delta$ denoting the symmetric difference (see 23 for more details). For a graph $G$ we define $\operatorname{CUTP}(G)$ to be the projection of $\operatorname{CUTP}\left(K_{n}\right)$ on the coordinates corresponding to the edges of the graph $G$. If $G$ is connected then $\operatorname{CUTP}(G)$ has exactly $2^{n-1}$ vertices. Then $\delta_{S}$ can be seen also as the adjacency matrix of a cut (into $S$ and $\bar{S}$ ) subgraph of $G$. The cut cone CUT $(G)$ is defined by taking the convex cone generated by the metrics $\delta_{S}$ but it is generally not used in that section.

In fact, $\operatorname{CUT}\left(K_{n}\right)$ is the set of all $n$-vertex semimetrics, which embed isometrically into some metric space $l_{1}$, and rational-valued elements of CUT $\left(K_{n}\right)$ correspond exactly to the $n$-vertex semimetrics, which embed isometrically, up to a scale $\lambda \in \mathbb{N}$, into the path metric of some $m$-cube $K_{2}^{m}$. It shows importance of this cone in Analysis and Combinatorics. The enumeration of orbits of facets of $\operatorname{CUT}\left(K_{n}\right)$ and $\operatorname{CUTP}\left(K_{n}\right)$ for $n \leq 7$ was done in [31, 3, 28, for $n=5,6,7$ respectively, and in [9], completed by [20, for $n=8$.

2.1. Automorphism group of cut polytopes. The symmetry group $\operatorname{Aut}(G)$ of a graph $G=(V, E)$ induces symmetry of $\operatorname{CUTP}(G)$. For any $U \subset\{1, \ldots, n\}$, the map $\delta_{S} \mapsto \delta_{U \Delta S}$ also defines a symmetry of $\operatorname{CUTP}(G)$. Together, those form the restricted symmetry group ARes $(\operatorname{CUTP}(G))$ of order $2^{|V|-1}|\operatorname{Aut}(G)|$. The full symmetry group $\operatorname{Aut}(\operatorname{CUTP}(G))$ may be larger. In Tables 10 , such cases are marked by *. Denote $2^{1-|V|}|\operatorname{Aut}(\operatorname{CUTP}(G))|$ by $A(G)$.

For example, $\left|\operatorname{Aut}\left(\operatorname{CUTP}\left(K_{n}\right)\right)\right|$ is $2^{n-1} n$ ! if $n \neq 4$ and $6 \times 2^{3} 4$ ! if $n=4$ ([13]).

Remark 1. (i) If $G=(V, E)$ is Prism $_{m}(m \neq 4)$, APrism $_{m}(m>3)$, Möbius ladder $M_{2 m}$ and $\operatorname{Pyr}^{2}\left(C_{m}\right)(m>3)$, then $\operatorname{Aut}(G)=4 m$.

(ii) If $G$ is a complete multipartite graph with $t_{1}$ parts of size $a_{1}, \ldots, t_{r}$ parts of size $a_{r}$, with $a_{1}<a_{2}<\cdots<a_{r}$ and all $t_{i} \geq 1$, then $\mid$ Aut $(G) \mid=\prod_{i=1}^{r} t_{i} !\left(a_{i} !\right)^{t_{i}}$. 
(iii) Among the cases considered here, all occurrences of $A(G)>|A u t(G)|$ are: $A(G)=m ! 2^{m-1}|A u t(G)|$ for $G=K_{2, m>2}, K_{1,1, m>1}$ and $A(G)=6|A u t(G)|$, i.e., $2 m !=48,6 m !$ for $G=K_{2,2}$ and $K_{1,1,1,1}$, respectively.

(iv) If $G=P_{m}(m \geq 3$ edges $)$, then $\mid$ Aut $(G)=2$, while $A(G)=m !=(|V|-1)$ !.

If $G=C_{m}(m>3)$, then $|A u t(G)|=2 m$, while $A(G)=2 m$ ! for $m=4$ and $A(G)=m !=|V|$ ! for $m \geq 5$.

\subsection{Edge faces, $s$-cycle faces and metric polytope.}

Definition 1. Let $G=(V, E)$ be a graph.

(i) Given an edge $e \in E$, the edge inequality (or 2-cycle inequality) is

$$
x(e) \geq 0 \text {. }
$$

(ii) Given a s-cycle $c=\left(v_{1}, \ldots, v_{s}\right), s \geq 3$, of $G$, the $s$-cycle inequality is:

$$
x\left(c,\left(v_{1}, v_{s}\right)\right)=\sum_{i=1}^{s-1} x\left(v_{i}, v_{i+1}\right)-x\left(v_{1}, v_{s}\right) \geq 0 .
$$

The edge inequalities and $s$-cycle inequalities are valid on $\operatorname{CUTP}(G)$, since they are, clearly, valid on each cut: a cut intersects a cycle in the set of even cardinality. So, they define faces, but not necessarily facets. In fact, it holds

Theorem 1. (i) The inequality $x(e)$ is facet defining in $\operatorname{CUTP}(G)$ (also, in $\operatorname{CUT}(G)$ ) if and only if $e$ is not contained into a 3-cycle of $G$.

(ii) An s-cycle inequality is facet defining in $\operatorname{CUTP}(G)$ (also, in $\mathrm{CUT}(G)$ ) if and only corresponding s-cycle is chordless.

(iii) $\operatorname{METP}(G)$ is defined by all edge and s-cycle inequalities, while $\operatorname{MET}(G)$ is defined by all s-cycle inequalities.

In fact, (i) and (ii) above were proved in 6, (iii) was proved in 5]; see also Section 27.3 in 23 .

The following Theorem, proved in 30 for cones and in 4 for polytopes, clarifies when the metric and cut polytope coincides:

Theorem 2. $\operatorname{CUT}(G)=\operatorname{MET}(G)$ or, equivalently, $\operatorname{CUTP}(G)=\operatorname{METP}(G)$ if and only if $G$ does not have any $K_{5}$-minor.

As a corollary of Theorem 2, we have that the facets of $\operatorname{CUTP}(G)$ (also, in $\operatorname{CUT}(G))$ are determined by edge inequalities and $s$-cycle inequalities if and only if $G$ does not have any $K_{5}$-minor.

3 -cycle inequality is usual triangle inequality; in fact, it is unique, among edge and all $s$-cycle inequalities to define a facet in a $\operatorname{CUTP}\left(K_{n}\right)$.

The girth and circumference of a graph, having cycles, are the length of the shortest and longest cycle, respectively. In a graph $G$, a chordless cycle is any cycle, which is induced subgraph; so, any triangle, any shortest cycle and any cycle, bounding a face in some embedding of $G$, are chordless. Let $c_{s}^{\prime}$ and $c_{s}$ denote the number of all and of all chordless $s$-cycles in $G$, respectively.

There are $2|E|$ edge faces, which decompose into orbits, one for each orbit of edges of $G$ under $\operatorname{Aut}(G)$. There are $2^{s-1} c_{s}^{\prime} s$-cycle faces, which decompose into orbits, one for each orbit of $s$-cycles of $G$ under $\operatorname{Aut}(G)$.

The incidence of edge faces is $2^{|V|-2}$ and the size of each orbit is twice the size of corresponding orbit of edges. The incidence of $s$-cycle faces is $2^{|V|-s} s$ and the size of each orbit is $2^{s-1}$ times the size of corresponding orbit of $s$-cycles in $G$. 
GENERALIZED CUT AND METRIC POLYTOPES OF GRAPHS AND SIMPLICIAL COMPLEXES

TABLE 1 . The number of facets of $\operatorname{CUTP}(G)$ of some $K_{5}$-minorfree graphs $G ;{ }^{*}$ shows $A(G)=2^{1-|V|}|\operatorname{Aut}(\operatorname{CUTP}(G))|>|A u t(G)|$

\begin{tabular}{||c|c||c|c|c||}
\hline \hline$G=(V, E)$ & $|V|,|E|$ & $A(G)$ & Number of facets & Orbit's $s$ \\
\hline \hline Möbius ladder $M_{8}$ & 8,2 & 16 & $184(4)$ & $2,2,4,5$ \\
$M_{6}=K_{3,3}$ & 6,9 & $2(3 !)^{2}$ & $90(2)$ & 2,4 \\
\hline$K_{1,1,1, m}, m>1$ & $m+3,3 m+3$ & $3 ! m !$ & $4+12 m(2)$ & 3,3 \\
$K_{1,2, m}, m>1$ & $m+3,3 m+2$ & $\left|A u t\left(K_{1,2, m}\right)\right|$ & $8 m+8\left(\begin{array}{c}m \\
2\end{array}\right)(2)$ & 3,4 \\
$K_{3, m}, m \geq 3$ & $m+3,3 m$ & $\left|A u t\left(K_{3, m}\right)\right|$ & $6 m+24\left(\begin{array}{c}m \\
2\end{array}\right)(2)$ & 2,4 \\
$K_{2, m}, m>2$ & $m+2,2 m$ & $2^{m-1} m !\left|A u t\left(K_{2, m}\right)\right|^{*}$ & $4 m^{2}(1)$ & 2 with 4 \\
$K_{2,2}$ & 4,4 & $6 \mid$ Aut $\left.\left(K_{2,2}\right)\right|^{*}$ & $16(1)$ & 2 with 4 \\
$K_{1,1, m}, m>1$ & $m+2,2 m+1$ & $2^{m-1} m !\left|A u t\left(K_{1,1, m}\right)\right|^{*}$ & $4 m(1)$ & 3 \\
$K_{m+1} K_{m}=K_{1, m}, m>1$ & $m+1, m$ & $m !$ & $2 m(1)$ & 2 \\
\hline APrism $m_{6}$ & 12,24 & 24 & $2,032(5)$ & $3,6,7,7,8$ \\
APrism $_{5}$ & 10,20 & 20 & $552(4)$ & $3,5,6,7$ \\
APrism $_{4}$ & 8,16 & 16 & $176(3)$ & $3,4,5$ \\
Prism $_{7}$ & 14,21 & 28 & $7,394(6)$ & $2,2,4,7,9,9$ \\
Prism $_{6}$ & 12,18 & 24 & $2,452(6)$ & $2,2,4,6,8,8$ \\
Prism $_{5}$ & 10,15 & 20 & $742(5)$ & $2,2,4,5,7$ \\
Prism $_{3}$ & 6,9 & 12 & $38(3)$ & $2,3,4$ \\
\hline Tr. Tetrahedron & 12,18 & 24 & $540(4)$ & $2,3,6,8$ \\
Cuboctahedron & 12,24 & 48 & $1,360(5)$ & $3,4,6,6,8$ \\
\hline Dodecahedron & 20,30 & 120 & $23,804(5)$ & $2,5,9,10,10$ \\
Icosahedron & 12,30 & 120 & $1,552(4)$ & $3,5,6,6$ \\
Cube $K_{2}^{2}$ & 8,12 & 48 & $200(3)$ & $2,4,6$ \\
Octahedron $K_{2,2,2}$ & 6,12 & 48 & $56(2)$ & 3,4 \\
Tetrahedron $K_{4}$ & 4,6 & $6 \mid$ Aut $\left(K_{4}\right) \mid *$ & $12(1)$ & 3 \\
\hline \hline
\end{tabular}

By Wagner's theorem [32, a finite graph is planar if and only if it has no minors $K_{5}$ and $K_{3,3}$. For embeddability on the projective plane $\mathbb{P}^{2}$, there are exactly 103 forbidden topological minors and exactly 35 forbidden minors (see 1, 27]). For embeddability on the torus $\mathbb{T}^{2}, 16629$ forbidden minors are known (see [26]) but the list is not necessarily complete. Closely related Kuratowski's theorem 29] states that a finite graph is planar if and only if it does not contain a subgraph that is a subdivision of $K_{5}$ or of $K_{3,3}$.

2.3. Skeletons of Platonic and semiregular polyhedra. Let $G$ be embedded in some oriented surface; so, it is a map $(V, E, F)$, where $F$ is the set of faces of $G$. Let $\vec{p}=\left(\ldots, p_{i}, \ldots\right)$ denote the $p$-vector of the map, enumerating the number $p_{i}>0$ of faces of all sizes $i$, existing in $G$.

Call face-bounding any $s$-cycle of $G$, bounding a face in map $G$. Call an $s$-cycle of $G i$-face-containing, edge-containing or point-containing, if all its interior points form just $i$-gonal face, edge or point, respectively. Call equator any cycle $C$, the interior of which (plus $C$ ) is isomorphic to the exterior (plus $C$ ).

The chordless 4, 6, 5, 9-cycles of Octahedron, Cube, Icosahedron and Dodecahedron, respectively, are exactly their vertex-containing 4, 6, 5, 9-cycles. 
TABLE 2. The number of facets of $\operatorname{CUTP}(G)$ for some graphs $G$ with $K_{5}$-minor

\begin{tabular}{|c|c|c|c|c|}
\hline 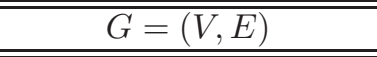 & $\overline{|| V|,| E \mid}$ & $A(G)$ & Number of facets (orbits) & Orbit's $s$ \\
\hline Heawood graph & 14,21 & 336 & $5,361,194(9)$ & $2,6,8$ \\
\hline Petersen graph & 10,15 & 120 & $3,614(4)$ & $2,5,6$ \\
\hline Möbius ladder $M_{10}$ & 10,15 & 20 & $1,414(5)$ & $2,2,4,6$ \\
\hline Möbius ladder $M_{12}$ & 12,18 & 24 & $26,452(6)$ & $2,2,4,7,9$ \\
\hline Möbius ladder $M_{14}$ & 14,21 & 28 & $369,506(9)$ & $2,2,4,8,10$ \\
\hline$K_{5,5}$ & 10,25 & $2(5 !)^{2}$ & $16,482,678,610(1,282)$ & 2,4 \\
\hline$K_{4,7}$ & 11,28 & $4 ! 7 !$ & $271,596,584(15)$ & 2,4 \\
\hline$K_{4,6}$ & 10,24 & $4 ! 6 !$ & $23,179,008(12)$ & 2,4 \\
\hline$K_{4,5}$ & 9,20 & $4 ! 5 !$ & $983,560(8)$ & 2,4 \\
\hline$K_{4,4}$ & 8,16 & $2(4 !)^{2}$ & $27,968(4)$ & 2,4 \\
\hline$K_{3,3,3}$ & 9,27 & $(3 !)^{4}$ & $624,406,788(2,015)$ & 3,4 \\
\hline$K_{1,4,4}$ & 9,24 & $2(4 !)^{2}$ & $36,391,264(175)$ & 3,4 \\
\hline$K_{1,3,5}$ & 9,23 & $3 ! 5 !$ & $71,340(7)$ & 3,4 \\
\hline$K_{1,3,4}$ & 8,19 & $3 ! 4 !$ & $12,480(6)$ & 3,4 \\
\hline$K_{1,3,3}$ & 7,15 & $2(3 !)^{2}$ & $684(3)$ & 3,4 \\
\hline$K_{1,1,3,3}$ & 8,21 & $4(3 !)^{2}$ & $432,552(50)$ & $3,3,4$ \\
\hline$K_{1,2,2,2}$ & 7,14 & $3 !(2 !)^{3}$ & $5,864(9)$ & $3,3,4$ \\
\hline$K_{1,1,2,2}$ & 6,13 & $4(2 !)^{2}$ & $184(4)$ & $3,3,4$ \\
\hline$K_{1,1,2, m}, m>2$ & $m+4,4 m+5$ & $4 m !$ & $8+20 m+8\left(\begin{array}{c}m \\
2\end{array}\right)(16 m-15)(7)$ & $3,3,3,4$ \\
\hline$K_{1,1,1,1, m}, m>1$ & $m+4,4 m+6$ & $4 ! m !$ & $8\left(8 m^{2}-3 m+2\right)(4)$ & 3,3 \\
\hline$K_{1,1,1,1,1,3}=K_{8}-K_{3}$ & 8.25 & 360 & $2,685,152(82)$ & 3,3 \\
\hline$K_{1,1,1,1,1,2}=K_{7}-K_{2}$ & 7,20 & 240 & $31,400(17)$ & 3,3 \\
\hline$K_{7}-C_{3}$ & 7,18 & 144 & $520(4)$ & 3,3 \\
\hline$K_{7}-C_{4}$ & 7,17 & 48 & $108(4)$ & $3,3,3$ \\
\hline$K_{7}-C_{5}=P y r^{2}\left(C_{5}\right)$ & 7,16 & 20 & $780(6)$ & $3,3,5$ \\
\hline$K_{7}-C_{6}=\operatorname{Pyr}\left(\right.$ Prism $\left._{3}\right)$ & 7,15 & 12 & $452(5)$ & $3,3,3,4$ \\
\hline$K_{7}-C_{7}$ & 7,14 & 14 & $148(3)$ & 3,4 \\
\hline Pyr $\left(\right.$ Prism $\left._{4}\right)$ & 9,20 & 48 & $10,464(6)$ & $3,4,6$ \\
\hline $\operatorname{Pyr}\left(\operatorname{Prism}_{5}\right)$ & 11,25 & 20 & $208,133(22)$ & $3,3,4,5,7$ \\
\hline Pyr $\left(\right.$ APrism $\left._{4}\right)$ & 9,24 & 16 & $389,104(17)$ & $3,3,3,4,5$ \\
\hline $\operatorname{Pyr}^{2}\left(C_{6}\right)$ & 8,19 & 24 & $3,432(7)$ & $3,3,6$ \\
\hline $\operatorname{Pyr}^{2}\left(C_{7}\right)$ & 9,22 & 28 & $14,740(11)$ & $3,3,7$ \\
\hline Tr.Octahedron on $\mathbb{P}^{2}$ & 12,18 & 48 & $62,140(7)$ & $2,2,4,6,6$ \\
\hline
\end{tabular}

For Octahedron and Cube, they are exactly all 3 and 4 equators, respectively, which are, apropos, the central circuits and zigzags (see [22]), respectively.

All $c_{6}$ chordless 6 -cycles of Icosahedron are exactly their 30 edge-containing ones and 10 face-containing ones, which are exactly the 10 equators and the weak zigzags ([22]). All $c_{10}$ chordless 10-cycles of Dodecahedron are 30 edge-containing ones and 6 face-containing ones, which are exactly all 6 equators and the zigzags.

Proposition 1. If $G$ is the skeleton of a Platonic solid, then all possible facets of $\operatorname{CUTP}(G)$ are: edge facets and s-cycle facets, coming from all face-bounding cycles 
and from all (if they exist and not listed before) vertex-, edge-, face-containing cycles.

For instance:

(i) If $G=K_{4}$ (Tetrahedron), then $\operatorname{CUTP}(G)$ has unique orbit of $2^{2} p_{3}=16$ (simplicial) 3-cycle facets (from all $|F|=p_{3}=4$ face-bounding cycles of $G$ ).

(ii) If $G=K_{2,2,2}$ (Octahedron), then $\operatorname{CUTP}(G)$ has 56 facets in 2 orbits, namely:

orbit of $2^{2} p_{3} 3$-cycle facets (from all $|F|=p_{3}=8$ face-bounding cycles, orbit of $2^{3} c_{4} 4$-cycle facets (from all $c_{4}=\frac{|V|}{2}=3$ vertex-containing 4-cycles).

(iii) If $G=K_{2}^{3}$ (Cube), then $\operatorname{CUTP}(G)$ has 200 facets in 3 orbits, namely: orbit of $2|E|=24$ edge facets, orbit of $2^{3} p_{4} 4$-cycle facets (from all $|F|=p_{4}=6$ face-bounding cycles),

orbit of $2^{5} c_{6}=1286$-cycle facets (from all $c_{6}=4$ vertex-containing 6 -cycles).

(iv) If $G$ is Icosahedron, then $\operatorname{CUTP}(G)$ has 1,552 facets in 4 orbits, namely: orbit of $2^{2} p_{3}=803$-cycle facets (from all $|F|=p_{3}=20$ face-bounding cycles), orbit of $2^{4} c_{5}=1925$-cycle facets (from all $c_{5}=12$ vertex-containing 5 -cycles), orbit of $2^{5}|E|=960$ 6-cycle facets (from $|E|=30$ edge-containing 6 -cycles), orbit of 320 6-cycle facets (from $\frac{|F|}{2}=10$ face-containing 6-cycles).

(v) If $G$ is Dodecahedron, then $\operatorname{CUTP}(G)$ has 23, 804 facets in 5 orbits, namely: orbit of $2|E|=60$ edge facets, orbit of $2^{4} p_{5}=192$ 5-cycle facets (from all $|F|=p_{5}=12$ face-bounding cycles), orbit of $2^{8} c_{9}=5,120$ 9-cycle facets (from all $c_{9}=20$ vertex-containing 9 -cycles), orbit of $2^{9}|E|=15,360$ 10-cycle facets (from 30 edge-containing 10 -cycles), orbit of $2^{9} \times 6=3,07210$-cycle facets (from $\frac{|F|}{2}=6$ face-containing 10-cycles).

In a Truncated Tetrahedron, call ring-edges those bounding a triangle, and rungedges all 6 other ones.

Proposition 2. (i) If $G$ is Truncated Tetrahedron, then $\operatorname{CUTP}(G)$ has 540 facets:

(1) orbit of $2 \times 6$ edge facets (from all 6 rung-edges),

(2) orbit of $2^{3} p_{3} 3$-cycle facets (from all $p_{3}=43$-face-bounding cycles),

(3) orbit of $2^{5} p_{6} 6$-cycle facets (from all $p_{6}=4$ 6-face-bounding cycles),

(4) orbit of $2^{7} \times 38$-cycle facets (from $\frac{1}{2}\left(\begin{array}{l}4 \\ 2\end{array}\right)$ rung-edge-containing 8 -cycles, which are also the equators).

(ii) If $G$ is Cuboctahedron, then $\operatorname{CUTP}(G)$ has 1,360 facets, namely:

(1) orbit of $2^{2} p_{3} 3$-cycle facets (from all $p_{3}=8$ 3-face-bounding cycles),

(2) orbit of $2^{3} p_{4} 4$-cycle facets (from all $p_{4}=6$ 4-face-bounding cycles),

(3) orbit of $2^{5}|V| 6$-cycle facets (from all 12 vertex-containing 6-cycles),

(4) orbit of $2^{5} \times 4=1286$-cycle facets (from all $\frac{p_{3}}{2} 3$-face-containing 6 -cycles, which are also equators and the central circuits),

(5) orbit of $2^{7} p_{4}=768$ 8-cycle facets (from all 6 4-face-containing 8-cycles, which are also zigzags).

Given a Prism $_{m}(m \neq 4)$ or an APrism $_{m}(m \neq 3)$, we call rung-edges the edges connecting two $m$-gons, and ring-edges other $2 m$ edges.

Let $P$ be an ordered partition $X_{1} \cup \cdots \cup X_{2 t}=\{1, \ldots, m\}$ into ordered sets $X_{i}$ of $\left|X_{i}\right| \geq 3$ consecutive integers. Call $P$-cycle of Prism $_{m}$ the chordless $(m+2 t)$-cycle obtained by taking the path $X_{1}$ on the, say, 1 -st $m$-gon, then rung edge (in the same direction, then path $X_{2}$ on the 2 -nd $m$-gon, etc. till returning to the path 
$X_{1}$. Any vertex of Prism $_{m}$ can be taken as the 1-st element of $X_{1}$, in order to fix a $P$-cycle. So, a $P$-cycle defines an orbit of $2^{m+2 t-1} 2 m(m+2 t)$-cycle facets of $\operatorname{CUTP}\left(\right.$ Prism $\left._{m}\right)$, except the case $\left(\left|X_{1}\right|, \ldots,\left|X_{m}\right|\right)=\left(\left|X_{2}\right|, \ldots,\left|X_{2 t}\right|,\left|X_{1}\right|\right)$ when the orbit is twice smaller.

A P-cycle of APrism $m$ is defined similarly, but we ask only $\left|X_{i}\right| \geq 2$ and rung edges, needed to change $m$-gon, should be selected, in the cases $\left|X_{i}\right|=2,3$ so that they not lead to a ring edge,i.e., a chord on $P$. Clearly, $P$-cycles are are all possible chordless $t$-cycles with $t \neq 4, m$ for Prism $m$ and with $t \neq 2, m$ for APrism $m$.

Proposition 3. (i) If $G$ is Prism $_{m}(m \geq 5)$, then all facets of $\left.\operatorname{CUTP}(G)\right)$ are:

(1) orbit of $2 m$ edge facets (from all $m$ rung-edges)

(2) orbit of $4 m$ edge facets (from all $2 m$ ring-edges);

(3) orbit of $2^{3} p_{4}=8 m$ 4-cycle facets (from all $m$ 4-face-bounding 4-cycles);

(4) orbit of $2^{m-1} p_{m}$ of $m$-cycle facets (from both $m$-face-bounding $m$-cycles);

(5) orbits of cycle facets for all possible P-cycles.

(ii) If $G$ is $\operatorname{APrism}_{m}(m \geq 4)$, then all facets of $\left.\operatorname{CUTP}(G)\right)$ are:

(1) orbit of $2^{2} p_{3}=8 m 3$-cycle facets (from all $2 m$ 3-face-bounding 3 -cycles);

(2) orbit of $2^{m-1} p_{m}$ of $m$-cycle facets (from both $m$-face-bounding $m$-cycles);

(3) orbits of cycle facets for all possible P-cycles.

2.4. Möbius ladders and Petersen graph. All Möbius ladders $M_{2 m}$ are toroidal. Möbius ladder $M_{6}=K_{3,3}$, Petersen graph and Heawood graph are both, toroidal and 1-planar.

Given the Möbius ladder $M_{2 m}$, call ring-edges $2 m$ those belonging to the $2 m$ cycle $C_{1, \ldots, 2 m}$, and rung-edges all other ones, i.e., $(i, i+m)$ for $i=1, \ldots, m$.

For any odd $t$ dividing $m$, denote by $C(m, t)$ the $(m+t)$-cycle of $M_{2 m}$, having, up to a cyclic shift, the form

$$
1, \ldots, 1+\frac{m}{t}, 1+\frac{m}{t}+m, \ldots, 1+\frac{2 m}{t}+m, 1+\frac{2 m}{t}+2 m, \ldots, 1+\frac{3 m}{t}+2 m, \ldots,
$$

i.e., $t$ consecutive sequences of $\frac{2 m}{t}-1$ ring-edges, followed by a rung-edge. Such $C(m, 1)$ exists for any $m \geq 3$; for $t>1$, their existence requires divisibility of $m$ by $t$. Clearly, the number of $(m+t)$-cycles $C(m, t)$ is $\frac{2 m}{t}$.

Conjecture 1. If $G=M_{2 m}$ ( $\left.m \geq 4\right)$, then among facets of $\operatorname{CUTP}(G)$ there are: two orbits of $4 m$ and $2 m$ edge facets (from all $2 m$ ring-and $m$ rung-edges), orbit of $2^{3} c_{4}=8 m 4$-cycles facets (from all $m$ 4-cycles), orbit of $2^{m} 2 m(m+1)$-cycle facets (from all $2 m(m+1)$-cycles $C(m, 1)$ ), for any odd divisor $t>1$ of $m$, orbit of $2^{m+t} \frac{m}{t}(m+t)$-cycle facets (from all $(m+t)$-cycles $C(m, t))$.

There are no other orbits for $m=3,4$ and for $m=3$ first two orbits unite into one of 18 edge facets, while all other orbits unite into one of $2^{3} c_{4}=724$-cycle facets. $\operatorname{CUTP}\left(M_{10}\right)$ has only one more orbit: the orbit of $2^{10}$ facets of incidence 15 (i.e., simplicial facets), defined by a cyclic shift of

$$
\sum_{i=1}^{10} \frac{1}{2}\left(3-(-1)^{i}\right) x_{i, i+1}+\sum_{i=0}^{m} x_{i, i+m}-2\left(x_{5,10}+2 x_{1,2}+x_{3,8}\right) .
$$

$\operatorname{CUTP}\left(M_{12}\right)$ also has only one more orbit: $2^{12} 6$ similar facets of incidence 20. 
Petersen graph has three circuit double covers: by six 5-gons (actually, zigzags), by five cycles of lengths $9,6,5,5,5$ and by 5 cycles of lengths $8,6,6,5,5$. It can be embedded in projective plane, in torus and in Klein bottle with corresponding sets of six, five and five faces.

Petersen graph have only $5-, 6-, 8-$ and 9 -cycles; it has $c_{5}=12$ and $c_{6}=10$. Heawood graph, i.e., $(3,6)$-cage, have the girth 6 and $c_{6}=28, c_{8}=|E|=21$.

Proposition 4. CUTP(Petersen graph) has 3, 614 facets in 4 orbits:

(1) orbit of $2|E|=30$ edge facets,

(2) orbit of $2^{4} c_{5}=1925$-cycle facets,

(3) orbit of $2^{5} c_{6}=3205$-cycle facets,

(4) orbit of $2^{10} 3$ simplexes, represented by

$$
\begin{aligned}
& \qquad\left(C_{12345}-2 x_{15}\right)-\left(C_{1^{\prime} 4^{\prime} 2^{\prime} 5^{\prime} 3^{\prime}}-x_{1^{\prime} 4^{\prime}}-x_{2^{\prime} 5^{\prime}}\right)+2 \sum_{1 \leq i \leq 5} x_{i i^{\prime}}, \\
& \text { where Petersen graph is seen as } C_{12345}+C_{1^{\prime} 4^{\prime} 2^{\prime} 5^{\prime} 3^{\prime}}+\sum_{1 \leq i \leq 5} x_{i i^{\prime}} .
\end{aligned}
$$

Remark 2. Three of all 9 orbits of facets of $\operatorname{CUTP}($ Heawood graph), are:

(1) $2|E|=42$ edge facets,

(2) $2^{5} c_{6}=8966$-cycle facets and

(3) $2^{7} c_{8}=2,688$ 8-cycle facets.

2.5. Complete-like graphs. $K_{n}$ is toroidal only for $n=5,6,7$, while it is 1 planar only for $n=5,6$. Among complete multipartite graphs $G$, the planar ones are: $K_{2, m} ; K_{1,1, m} ; K_{1,2,2} ; K_{1,1,1,1}=K_{4}$ and their subgraphs. The 1-planar $G$ are, besides above: $K_{6} ; K_{1,1,1,6} ; K_{1,1,2,3} ; K_{2,2,2,2} ; K_{1,1,1,2,2}$ and their subgraphs ([11])

Given sets $A_{1}, \ldots, A_{t}$ with $t \geq 2$ and $1 \leq\left|A_{1}\right| \leq \cdots \leq\left|A_{t}\right|$, let $G$ be complete multipartite graph $K_{a_{1}, \ldots, a_{t}}$ with $a_{i}=\left|A_{i}\right|$ for $1 \leq i \leq t$.

All possible chordless cycles in $G$ are $c_{3}=\sum_{1<i<j<k<t} a_{i} a_{j} a_{k}$. triangles and $c_{4}=\sum_{1 \leq i \leq t}\left(\begin{array}{c}a_{i} \\ 2\end{array}\right)\left(\begin{array}{c}a_{j} \\ 2\end{array}\right)$ quadrangles. Hence, $c_{3}>0$ if and only if $t>2$ and $c_{4}>0$ if and only if $\left(a_{1}, t\right) \neq(1,2)$. So, among edge and $s$-cycle facets of $\operatorname{CUTP}(G)$, only three such orbits are possible: $2|E|$ edge facets if $t=2,4 c_{3} 3$-cycle facets if $\geq 3$ and $8 c_{4}$ 4-cycle facets if $\left(a_{1}, t\right) \neq(1,2)$.

All cases, when there are no other facets, i.e., when $G$ has no $K_{5}$-minor, are given in Table 1) note that the facets are simplexes for $G=K_{2,2}$ and $K_{1,1,1,1}$. In particular, $G=K_{m+i}-K_{m}, m>1$, has no $K_{5}$-minor only for $i=1,2,3$. The facets of $\operatorname{CUTP}(G)$ are the orbit of $2 m$ edge facets for $i=1$, the orbit of $2 m 3$-cycle facets for $i=2$ and two orbits (of sizes $12 m$ and 4 ) of 3-cycle facets for $i=3$.

Some of remaining cases presented in Table 2. For $G=K_{m+4}-K_{m}=K_{1,1,1,1, m>1}$ and $K_{1,1,2, m>2}$, the number of orbits stays constant for any $m$ : 4 and 7 , respectively.

Given sequence $b_{1}, \ldots, b_{n}$ of integers, which sum to 1 , let us call

$$
\operatorname{hyp}(b)=\sum_{1 \leq i, j \leq n} x_{i j} b_{i} b_{j} \leq 0
$$

(when it is applicable) hypermetric inequality. Note that hyp $(1,1,-1,0, \ldots, 0)$ is usual triangle inequality. Denote $h y p(b)$ with all non-zero $b_{i}$ being $b_{x}=b_{y}=1=$ $-b_{z}$ by $\operatorname{Tr}(x, y ; z)$ and $h y p(b)$ with all non-zero $b_{i}$ being $b_{x}=b_{y}=b_{z}=1=-b_{u}=$ -bv by $\operatorname{Pent}(x, y, z ; u, v)$.

If $G=K_{1,1,2, m}$ with $m \geq 3$, then $\operatorname{CUTP}(G)$ has $8+20 m+8\left(\begin{array}{c}m \\ 2\end{array}\right)(16 m-15)$ facets in 7 orbits: 3 orbits of $8,4 m, 16 m$ 3-cycle facets, one orbit of $8\left(\begin{array}{c}m \\ 2\end{array}\right)$ 4-cycle facets and 
3 orbits of $64\left(\begin{array}{c}m \\ 2\end{array}\right), 64\left(\begin{array}{c}m \\ 2\end{array}\right), 384\left(\begin{array}{c}m \\ 3\end{array}\right)\{0, \pm 1\}$-valued non-s-cycle facets, having 4 values -1 and $11,11,12$ values of 1 . The partition is $\{1\},\{2\},\{3,4\},\{5, \ldots, m+4\}$.

$\operatorname{CUTP}\left(K_{1,1,2,2}\right)$ has 184 facets in 4 orbits: 2 orbits of $8+8,323$-cycle facets, one orbit of 84 -cycle facets and one orbits of $2^{7}$ facets, represented by

$$
\operatorname{hyp}(1,1,1,-1,-1,0)+\operatorname{hyp}(0,0,1,1,0,-0,-1) \leq 0 .
$$

The graph $G=K_{m+t}-K_{m}=K_{1, \ldots, 1, m}$ has a $K_{5}$-minor only if $t \geq 4$. If $m \geq 3$, then $\operatorname{CUTP}(G)$ has 2 orbits of $4 m\left(\begin{array}{l}t \\ 2\end{array}\right)$ and $4\left(\begin{array}{l}t \\ 3\end{array}\right)$ 3-cycle facets and, for $t<4$ only, no other facets. The partition is $\{1\}, \ldots,\{t\},\{t+1, \ldots, t+m\}$.

If $G=K_{m+4}-K_{m}$, then $\operatorname{CUTP}(G)$ has $8\left(8 m^{2}-3 m+2\right)$ facets in 4 orbits: 2 orbits of $24 m, 163$-cycle facets and 2 orbits of sizes $16 m, 128\left(\begin{array}{c}m \\ 2\end{array}\right)$, represented by $\operatorname{hyp}(1,1,-1,-1,1,0, \ldots, 0) \leq 0$, i.e., Pent $(1,2,5 ; 3,4)$ and

$$
\operatorname{hyp}(1,1,-1,0,1,-1,0, \ldots, 0)+\operatorname{hyp}(0,0,0,-1,1,1,0, \ldots, 0) \leq 0 .
$$

If $G=K_{m+5}-K_{m}$, then among many orbits of facets of $\operatorname{CUTP}(G)$, there are 2 orbits of $40,40 m$ 3-cycle facets and 3 orbits of $16,80 m, 20 m(m-1)$ facets, represented, respectively, by

(1) $\operatorname{hyp}(1,1,1,-1,-1,0, \ldots, 0) \leq 0$,

(2) $\operatorname{hyp}(1,1,-1,-1,0,1,0, \ldots, 0) \leq 0$

(3) and $h y p(1,-1,-1,0,0,1,1,0, \ldots, 0)+h y p(0,0,0,1,0,1-1,0, \ldots, 0) \leq 0$.

Among 12 remaining orbits for $K_{7}-K_{2}$, two (of sizes $2^{7} 30,2^{7} 60$ ) are $\{0, \pm 1\}$ valued; they are represented, respectively, by

(1) $\operatorname{hyp}(1,1,-1,-1,1,1,-1)+\left(x_{34}+x_{47}-x_{2,7}+x_{12}-x_{13}\right) \leq 0$ and

(2) $\left(x_{13}+x_{34}+x_{45}+x_{15}\right)+\left(x_{23}+x_{36}+x_{67}+x_{27}\right)-\left(x_{14}+x_{47}-x_{57}+x_{25}+\right.$ $\left.x_{26}+x_{16}\right)$.

Let $G=\operatorname{Pyr}^{2}\left(C_{m}\right)$. Clearly, it is $K_{4}, K_{5}$ if $m=2,3$, respectively. For $m \geq 4$, it hold $A(G)=4 m$ and all chordless cycles $3 m$ triangles and unique $m$-cycle. Any of $3 m+1$ edges belongs to a triangle. So, among orbits of facets of $\operatorname{CUTP}(G)$, there are two (of size $8 m$ and $4 m$ ) orbits of 3 -cycle facets and orbit of $2^{m-1} m$-cycle facets. All other facets for $m \leq 7$ are $\{0, \pm 1\}$-valued.

For $\operatorname{Pyr}^{2}\left(C_{1 \ldots m}\right)$ with $m=4$, unique remaining orbit consists of $2^{7}$ facets, represented by $\operatorname{Pent}(3,5,5 ; 1,2)+\operatorname{Tr}(1,2 ; 4)$. Among remaining orbits for $m=5$ and 7 , there is an orbit of $2^{m+1}$ facets represented by

(1) $\operatorname{Pyr}^{2}\left(C_{12345}\right)-2\left(\left(x_{45}+x_{67}\right)+\left(x_{16}+x_{17}+x_{36}+x_{37}\right)\right) \leq 0$ and, respectively, by

(2) $\operatorname{Pyr}^{2}\left(C_{12345}\right)-2\left(\left(x_{12}+x_{19}\right)+\left(x_{29}+x_{38}+x_{49}+x_{58} x_{69}+x_{78}\right)\right) \leq 0$.

For $m=5$, two remaining orbits (each of size $2^{6} 5$ ) are represented by

(1) $C_{12345}-2 x_{15}+x_{67}+\left(\left(x_{17}-x_{16}\right)-\left(x_{37}-x_{36}\right)+\left(x_{47}-x_{46}\right)\right) \leq 0$ and

(2) $C_{12345}-2 x_{15}+x_{67}+\left(\left(x_{17}-x_{16}\right)-\left(x_{47}-x_{46}\right)+\left(x_{57}-x_{56}\right)\right) \leq 0$, respectively.

For $m=6$, one of 4 remaining orbits (of size $2^{7} 6$ ) is represented by

$C_{123456}-2 x_{12}+x_{78}+\left(\left(x_{17}-x_{18}\right)+\left(x_{57}-x_{58}\right)-\left(x_{67}-x_{68}\right)\right) \leq 0$.

Note that $K_{7}-C_{5}=\operatorname{Pyr}^{2}\left(C_{5}\right)$. Now, $G=K_{7}-C_{1234}=K_{\{7\},\{6\},\{5\},\{1,3\},\{2,4\}}$ has $c_{3}=19 ; \operatorname{CUTP}(G)$ has four orbits of facets: three (of sizes 48,24,4) of 3 -cycle facets and one orbit of size 32 , represented by $\operatorname{Pent}(4,5,6 ; 2,7)$. Each of $K_{5}$-minors, $K_{\{2,4,5,6,7\}}$ and $K_{\{1,3,5,6,7\}}$ provides 16 of above 32 facets. 
$G=K_{7}-C_{7}$ has $c_{3}=c_{4}=7 ; \operatorname{CUTP}(G)$ has three orbits of facets: one (of size 28) of 3-cycle facets, one (of size 56) of 4-cycle facets and one of size 64, represented by $\left(K_{7}-C_{1234567}\right)-2\left(x_{15}+\right.$ Path $\left._{27364}\right)$.

\section{QUASI-METRIC POLYTOPES OVER GRAPHS}

We first define the inequalities satisfied by quasi-metrics on $n$-points.

Definition 2. Given a fixed $n \geq 3$ we define:

(i) The oriented triangle inequality for all $1 \leq i, j, k \leq n$

$$
d(i, j) \leq d(i, k)+d(k, j)
$$

(ii) The non-negativity inequality for all $1 \leq i, j \leq n$ is

$$
d(i, j) \geq 0
$$

(iii) A bounded oriented metric is a metric satisfying for all $1 \leq i, j, k \leq n$ the inequalities

$$
d(j, i)+d(i, k)+d(k, j) \leq 2 \text { and } d(i, j) \leq 1 .
$$

Using this we can define the cone of quasimetrics QMET $\left(K_{n}\right)$ (see [19, 16] for more details) to be the cone of oriented metrics satisfying the inequalities (i), (ii) of 2. We define the polytope $\operatorname{QMETP}\left(K_{n}\right)$ to be the set of metrics satisfying the inequalities of 2

Given a subset $S \subset\{1, \ldots, n\}$ we define the oriented switching:

$$
F_{S}(d)(i, j)=\left\{\begin{array}{cl}
1-d(j, i) & \text { if }|S \cap\{i, j\}|=1 \\
d(i, j) & \text { otherwise. }
\end{array}\right.
$$

The symmetric group $\operatorname{Sym}(n)$ acts on $\operatorname{QMET}\left(K_{n}\right)$ and define a group of size $n$ !. The oriented switchings determine and $\operatorname{Sym}(n)$ act on $\operatorname{QMETP}\left(K_{n}\right)$ and determine a group of size $2^{n-1} n$ !.

The cone $\operatorname{MET}\left(K_{n}\right)$ and polytope $\operatorname{METP}\left(K_{n}\right)$ are embedded into $\operatorname{QMET}\left(K_{n}\right)$ and $\operatorname{QMETP}\left(K_{n}\right)$ but we have another interesting subset:

Definition 3. Given $n \geq 3$ and an oriented metric $d \in \operatorname{QMET}\left(K_{n}\right)$, $d$ is called weightable if it satisfies the following equivalent definitions:

(i) An oriented metric is called weightable if there exist a function $w_{i}$ such that for all $1 \leq i, j \leq n$

$$
d(i, j)+w_{i}=d(j, i)+w_{j}
$$

(ii) For all $1 \leq i, j, k \leq n$ we have

$$
d(i, j)+d(j, k)+d(k, i)=d(j, i)+d(k, j)+d(i, k)
$$

We thus define the cone $\operatorname{WQMET}\left(K_{n}\right)$ and polytope $\operatorname{WQMETP}\left(K_{n}\right)$ to be the set of weightable quasimetrics of the cone $\operatorname{QMET}\left(K_{n}\right)$ and polytope $\operatorname{QMETP}\left(K_{n}\right)$. Clearly, the oriented switching preserves $\operatorname{WQMETP}\left(K_{n}\right)$.

With all those definitions we can now define the corresponding objects on graphs:

Definition 4. Let $G$ be an undirected graph; we define $E(G)$ the set of edges and $\operatorname{Dir}(E(G))$ to be the set of directed edges of $G$ :

(i) We define the cones $\operatorname{QMET}(G)$ and $\operatorname{WQMET}(G)$ to be the projections of the cones $\operatorname{QMET}\left(K_{n}\right)$ and $\operatorname{WQMET}\left(K_{n}\right)$ on $\mathbb{R}^{\operatorname{Dir}(E(G))}$.

(ii) We define the polytopes $\operatorname{QMETP}(G)$ and $\operatorname{WQMETP}(G)$ to be the projections of the polytopes $\operatorname{QMETP}\left(K_{n}\right)$ and $\operatorname{WQMETP}\left(K_{n}\right)$ on $\mathbb{R}^{\operatorname{Dir}(E(G))}$. 
We can now give a description by inequalities of $\operatorname{QMET}(G)$ :

Theorem 3. For a given graph $G$ the polyhedral cone $\operatorname{QMET}(G)$ is defined as the set of functions $\mathbb{R}^{\operatorname{Dir}(E)}$ such that

(i) For any directed edge $e=(i, j)$ of $G$ the inequality $0 \leq d(i, j)$.

(ii) For any oriented cycle $e=\left(v_{1}, v_{2}, \ldots, v_{m}\right)$ of $G$

$$
d\left(v_{1}, v_{m}\right) \leq d\left(v_{1}, v_{2}\right)+d\left(v_{2}, v_{3}\right)+\cdots+d\left(v_{m-1}, v_{m}\right)
$$

The same results holds for $\mathrm{WQMET}(G)$ by adding the extra condition that there exist a function $w$ such that $d(i, j)-d(j, i)=w_{i}-w_{j}$.

Proof. Our proof is adapted from the proof of [23, Theorem 27.3.3]. It is clear that the cycle inequalities (i) and (ii) are valid for $d \in \operatorname{QMET}\left(K_{n}\right)$ and that edges of $G$ do not occur in their expression. Therefore, the inequalities are also valid for the projection.

The proof of sufficiency is done by induction and is more complicated. Suppose that the result is proved for $G+e$, i.e. $G$ to which an edge $e=(i, j)$ has been added. Suppose we have an element $x$ of $\mathbb{R}^{\operatorname{Dir}(E(G))}$ satisfying all oriented cycle inequalities.

We need to find an antecedent of $x$, i.e. a function $y \in \mathbb{R}^{\operatorname{Dir}(E(G)+e)}$. That is we need to find $y(i, j)$ and $y(j, i)$.

We write $P_{i, j}$ to be the set of directed paths from $i$ to $j$ in $G$. Assume first that $P_{i, j} \neq \emptyset$. We write

$$
u_{i, j}=\min _{u \in P_{i, j}} x(u)
$$

since $x$ is non-negative, we have $u_{i, j} \geq 0$. We then write

$$
l_{i, j}=\max _{v \in P_{i, j}, f \in v} x(r(f))-x(v-f)
$$

with $r(f)$ the reversal of the directed edge $f$. If $P_{i, j}=\emptyset$, i.e. if the edge $e$ is connecting two connected components of $G$ then we set $l_{i, j}=u_{i, j}=0$.

We have $l_{i, j} \leq u_{i, j}$ since otherwise we could take a path $u$ realizing the minimum $u_{i, j}$, a path $v$ and directed edge $f$ realizing the maximum $l_{i, j}$ put it together and get a counterexample to the oriented cycle inequality (ii).

So, we can find a value $y_{i, j}$ such that

$$
l_{i, j} \leq y_{i, j} \leq u_{i, j}
$$

and since $u_{i, j} \geq 0$ we can choose $y_{i, j} \geq 0$. The same holds for $y_{j, i}$. Therefore we found an antecedent of $x$ in $\mathbb{R}^{\operatorname{Dir}(E(G)+e)}$ and this proves the result for $\operatorname{QMET}(G)$ and so the stated theorem.

For WQMET $(G)$ we have to adjust the induction construction. If $P_{i, j}=\emptyset$ then we can adjust the values of the weights $w$ such that $w_{i}=w_{j}$. This is possible since the weights are determined up to a constant term.

On the other hand if $P_{i, j}$ is not empty then the weight is already given and we should get in the end $y_{i, j}-y_{j, i}=w_{i}-w_{j}$. Actually this is not a problem since it can be easily be shown that $u_{i, j}-u_{j, i}=w_{i}-w_{j}$ and $l_{i, j}-l_{j, i}=w_{i}-w_{j}$ and so the inductive construction works.

Now we turn to the construction for the polytope case.

Theorem 4. For a given graph $G$ the polytope $\operatorname{QMETP}(G)$ is defined as the set of functions $\mathbb{R}^{\text {Dir(E) }}$ such that

(i) For any directed edge $e=(i, j)$ of $G$ the inequality $0 \leq d(i, j) \leq 1$ holds 
(ii) For any oriented cycle $C=\left(v_{1}, v_{2}, \ldots, v_{m}\right)$ of $G$ and subset $F$ of odd size

$$
\sum_{f=\left(v, v^{\prime}\right) \in F} d\left(v^{\prime}, v\right)-\sum_{f=\left(v, v^{\prime}\right) \in C-F} d\left(v, v^{\prime}\right) \leq|F|-1
$$

The same results holds for $\operatorname{WQMETP}(G)$ with the extra condition that there exist a function $w$ such that $d(i, j)-d(j, i)=w_{i}-w_{j}$.

Proof. The proof follows by remarking that the inequalities (i) and (ii) are the oriented switchings of the non-negative inequality and oriented cycle inequality 2 Thus the proof follow from Theorem 3 and the same proof strategy as 23 , Theorem 27.3.3].

The oriented multicut cones defined in the introduction are very complicated. In particular the oriented multicuts are not stable under oriented switchings. However, we have $\operatorname{WOMCUTP}\left(K_{n}\right)=\operatorname{WQMETP}\left(K_{n}\right)$ for $n \leq 4$. Based on that and analogy with Theorem 2 a natural conjecture would be that $\operatorname{WOMCUTP}(G)=$ WQMETP $(G)$ if $G$ has no $K_{5}$ minor. But it seems that for some other graphs with no $K_{5}$ minor we have $\operatorname{WOMCUTP}(G) \neq \operatorname{WQMETP}(G)$.

\section{HEMI-METRIC POLYTOPES OVER SIMPLICIAL COMPLEXES}

We can also generate metrics to a measure of distance of more than 2 objects. Our approach differs from [15, 14, 17, 21] and has the advantage of allowing to define it on complexes.

We consider by $\operatorname{Set}_{n, m}$ the set of subsets of $m+1$ points of $\{1, \ldots, n\}$.

Definition 5. Let us fix $m \geq 1$ and $n$ :

(i) A m-dimensional complex is formed by a subset of Set $t_{n, m}$.

(ii) A closed manifold of dimension $m$ is formed by a subset $\mathcal{S}$ of Set $_{n, m}$ such that for each subset $S$ of $m$ points of $\{1, \ldots, n\}$ the number of simplices of $\mathcal{S}$ containing $S$ is even.

For the case $m=1$ the closed manifold of above definition corresponds to the closed cycles. We now proceed to defining the corresponding cycle inequalities:

Definition 6. Let us fix $m \geq 1$ and $n$. Given a m-dimensional complex $K$ on $\{1, \ldots, n\}$, the hemimetric cone $\operatorname{HMET}(K)$ is formed by the functions $d$ on $K$ satisfying

(i) the non-negative inequalities

$$
d(\Delta) \geq 0
$$

for all $\Delta \in K$.

(ii) For all closed manifolds $\left(\Delta_{1}, \ldots, \Delta_{r}\right)$ formed by simplices $\Delta_{i} \in K$ the inequalities

$$
d\left(\Delta_{i}\right) \leq \sum_{1 \leq j \leq r, i \neq j} d\left(\Delta_{j}\right)
$$

for all $1 \leq i \leq r$.

For $m=1$ the definition corresponds to the one of $\operatorname{MET}(G)$.

Theorem 5. Let us fix $m \geq 1$ and $n$. Let us take $K$ a m-dimensional complex on $n$ points. The cone $\operatorname{HMET}(K)$ is the projection of $\operatorname{HMET}\left(S_{e} t_{n, m}\right)$ on the simplices included in $K$. 
Proof. Our proof is adapted from the proof for metric of [23, Theorem 27.3.3]. The inequalities for $\operatorname{HMET}(K)$ are clearly valid on $\operatorname{HMET}\left(\operatorname{Set}_{n, m}\right)$ which proves one inclusion.

We want to prove it by induction the other inclusion. Suppose that we have a metric $d \in \operatorname{HMET}(K)$ and a simplex $\Delta \notin K$. We want to find a metric $d^{\prime}$ on $\operatorname{HMET}(K+\Delta)$. That is we need to find a value of $d(\Delta)$ that extends the inequality. For a subset $S \subset S e t_{n, m}$ we define

$$
d(S)=\sum_{\Delta^{\prime} \in S} d\left(\Delta^{\prime}\right)
$$

Let us consider the

$$
W_{K, \Delta}=\{U \subset K: U \cup\{\Delta\} \text { is a closed manifold }\} .
$$

We now define the upper bound

$$
u_{K, \Delta}=\min _{U \in W_{K, \Delta}} d(U) .
$$

We have $u_{K, \Delta} \geq 0$ since $d \in \operatorname{HMET}(K)$ implies $d\left(\Delta^{\prime}\right) \geq 0$.

The lower bound is formed by

$$
l_{K, \Delta}=\max _{P \in W_{K, \Delta}, F \in P} d(F)-d(P-F) .
$$

Suppose that $l_{K, \Delta}>u_{K, \Delta}$. We have $u_{K, \Delta}$ realized by $U_{0}$ and $l_{K, \Delta}$ is realized by $L_{0}$ and a face $F_{0} \in L_{0}$. The union $L_{0} \cup U_{0}$ is not necessarily a closed manifold since $L_{0} \cup U_{0}$ may share simplices. If that is so we remove them and consider instead $W_{0}=L_{0} \cup U_{0}-L_{0} \cap U_{0}$.

The inequality $l_{K, \Delta}>u_{K, \Delta}$ implies then

$$
d\left(F_{0}\right)>d\left(L_{0}-F_{0}\right)+d\left(U_{0}\right)=d\left(W_{0}-F_{0}\right)+2 d\left(L_{0} \cap U_{0}\right) \geq d\left(W_{0}-F_{0}\right)
$$

which violates the fact that $d \in \operatorname{HMET}(K)$. Thus we can find a value $\alpha$ with

$$
l_{K, \Delta} \leq \alpha \leq u_{K, \Delta} \text { and } \alpha \geq 0 .
$$

Thus we can find a value for $d(\Delta)$ that is compatible with an extension.

The inequality set defining $\operatorname{HMET}(K)$ is highly redundant but is still finite so, the cone $\operatorname{HMET}(K)$ is actually polyhedral.

On the other hand, using the inequalities obtained from the simplex does not work. Consider for example the complex $\operatorname{Set}_{6,2}$. The Octahedron has 6 vertices and 8 faces and is a closed manifold. Thus it determines an inequality of the form

$$
x_{000} \leq x_{100}+x_{010}+x_{001}+x_{110}+x_{101}+x_{011}+x_{111}
$$

which is not implied by the inequality on the simplices. The proof can be done by linear programming using our software polyhedral (25). This proves that our construction is different from the one of [15, 14, 21] and it would be interesting to redo the computations of those works.

\section{Acknowledgments}

Second author gratefully acknowledges support from the Alexander von Humboldt foundation. 
GENERALIZED CUT AND METRIC POLYTOPES OF GRAPHS AND SIMPLICIAL COMPLEXES

\section{REFERENCES}

[1] D. Archdeacon, A Kuratowski theorem for the projective plane, J. Graph Theory 5 (1981), no. 3, 243-246, URL: http://dx.doi.org/10.1002/jgt.3190050305 doi:10.1002/jgt.3190050305.

[2] D. Avis, P. Hayden, and M. M. Wilde, Leggett-Garg inequalities and the geometry of the cut polytope, Phys. Rev. A (3) $\mathbf{8 2}$ (2010), no. 3, 030102, 4, URL: http://dx.doi.org/10.1103/PhysRevA.82.030102 doi:10.1103/PhysRevA.82.030102.

[3] D. Avis and Mutt, All the facets of the six-point Hamming cone, European J. Combin. 10 (1989), no. 4, 309-312, URL: http://dx.doi.org/10.1016/S0195-6698(89)80002-2, doi:10.1016/S0195-6698(89)80002-2.

[4] F. Barahona, The max-cut problem on graphs not contractible to $K_{s}$, Oper. Res. Lett. 2 (1983), no. 3, 107-111, URL: http://dx.doi.org/10.1016/0167-6377(83)90016-0, doi:10.1016/0167-6377(83)90016-0.

[5] F. Barahona, On cuts and matchings in planar graphs, Math. Programming 60 (1993), no. 1, Ser. A, 53-68, URL: http://dx.doi.org/10.1007/BF01580600, doi:10.1007/BF01580600.

[6] F. Barahona and A. R. Mahjoub, On the cut polytope, Math. Programming 36 (1986), no. 2, 157-173, URL: http://dx.doi.org/10.1007/BF02592023, doi:10.1007/BF02592023.

[7] D. Bryant and P. F. Tupper, Hyperconvexity and tight-span theory for diversities, Adv. Math. 231 (2012), no. 6, 3172-3198, URL: http://dx.doi.org/10.1016/j.aim.2012.08.008, doi:10.1016/j.aim.2012.08.008.

[8] D. Bryant and P. F. Tupper, Diversities and the geometry of hypergraphs, Discrete Math. Theor. Comput. Sci. 16 (2014), no. 2, 1-20.

[9] T. Christof and G. Reinelt, Decomposition and parallelization techniques for enumerating the facets of combinatorial polytopes, Internat. J. Comput. Geom. Appl. 11 (2001), no. 4, 423437, URL: http://dx.doi.org/10.1142/S0218195901000560 doi:10.1142/S0218195901000560.

[10] P. Crescenzi and V. Kann, Approximation on the web: a compendium of NP optimization problems, Randomization and approximation techniques in computer science (Bologna, 1997), Lecture Notes in Comput. Sci., vol. 1269, Springer, Berlin, 1997, pp. 111-118, URL: http://dx.doi.org/10.1007/3-540-63248-4_10 doi:10.1007/3-540-63248-4_10.

[11] J. Czap and D. Hudák, 1-planarity of complete multipartite graphs, Discrete Appl. Math. 160 (2012), no. 4-5, 505-512, URL: http://dx.doi.org/10.1016/j.dam.2011.11.014, doi:10.1016/j.dam.2011.11.014.

[12] E. Deza, M. Deza, and M. Dutour Sikirić, Generalizations of finite metrics and cuts, World Scientific Publishing Co. Pte. Ltd., Hackensack, NJ, 2016, URL: http://dx.doi.org/10.1142/9906 doi:10.1142/9906.

[13] M. Deza, V. P. Grishukhin, and M. Laurent, The symmetries of the cut polytope and of some relatives, Applied geometry and discrete mathematics, DIMACS Ser. Discrete Math. Theoret. Comput. Sci., vol. 4, Amer. Math. Soc., Providence, RI, 1991, pp. 205-220.

[14] M.-M. Deza and I. G. Rosenberg, n-semimetrics, European J. Combin. 21 (2000), no. 6, 797806, Discrete metric spaces (Marseille, 1998), URL: http://dx.doi.org/10.1006/eujc.1999.0384. doi:10.1006/eujc.1999.0384.

[15] M.-M. Deza and I. G. Rosenberg, Small cones of m-hemimetrics, Discrete Math. 291 (2005), no. 1-3, 81-97, URL: http://dx.doi.org/10.1016/j.disc.2004.04.022 doi:10.1016/j.disc.2004.04.022.

[16] M. Deza, M. Dutour, and E. Panteleeva, Small cones of oriented semi-metrics, Forum for Interdisciplinary Mathematics Proceedings on Statistics, Combinatorics \& Related Areas (Bombay, 2000), vol. 22, 2002, pp. 199-225, URL: http://dx.doi.org/10.1080/01966324.2002.10737587, doi:10.1080/01966324.2002.10737587.

[17] M. Deza, M. Dutour, and H. Maehara, On volume-measure as hemi-metrics, Ryukyu Math. J. 17 (2004), 1-9.

[18] M. Deza and M. Dutour Sikirić, The hypermetric cone and polytope on eight vertices and some generalizations, J. Symb. Comp. to appear (2017).

[19] M. Deza and E. Panteleeva, Quasi-semi-metrics, oriented multi-cuts and related polyhedra, European J. Combin. 21 (2000), no. 6, 777-795, Discrete metric spaces (Marseille, 1998), URL: http://dx.doi.org/10.1006/eujc.1999.0383 doi:10.1006/eujc.1999.0383.

[20] M. Deza and M. D. Sikirić, Enumeration of the facets of cut polytopes over some highly symmetric graphs, Int. Trans. Oper. Res. 23 (2016), no. 5, 853-860, URL: http://dx.doi.org/10.1111/itor.12194, doi:10.1111/itor.12194. 
[21] M.-M. Deza and M. Dutour, Cones of metrics, hemi-metrics and super-metrics, Ann. Eur. Acad. Sci. 1 (2003), 141-162.

[22] M.-M. Deza, M. Dutour Sikirić, and M. I. Shtogrin, Geometric structure of chemistry-relevant graphs, Forum for Interdisciplinary Mathematics, vol. 1, Springer, New Delhi, 2015, Zigzags and central circuits, URL: http://dx.doi.org/10.1007/978-81-322-2449-5, doi:10.1007/978-81$322-2449-5$.

[23] M. M. Deza and M. Laurent, Geometry of cuts and metrics, Algorithms and Combinatorics, vol. 15, Springer, Heidelberg, 2010, First softcover printing of the 1997 original [MR1460488], URL: http://dx.doi.org/10.1007/978-3-642-04295-9 doi:10.1007/978-3-642-04295-9.

[24] M. Dutour Sikirić, Cut polytopes, URL: http://mathieudutour.altervista.org/CutPolytopes/

[25] M. Dutour Sikirić, Polyhedral, URL: http://mathieudutour.altervista.org/Polyhedral/

[26] A. Gagarin, W. Myrvold, and J. Chambers, The obstructions for toroidal graphs with no $K_{3,3}$ 's, Discrete Math. 309 (2009), no. 11, 3625-3631, URL: http://dx.doi.org/10.1016/j.disc.2007.12.075 doi:10.1016/j.disc.2007.12.075.

[27] H. H. Glover, J. P. Huneke, and C. S. Wang, 103 graphs that are irreducible for the projective plane, J. Combin. Theory Ser. B 27 (1979), no. 3, 332-370, URL: http://dx.doi.org/10.1016/0095-8956(79)90022-4, doi:10.1016/0095-8956(79)90022-4.

[28] V. P. Grishukhin, All facets of the cut cone $\mathbf{C}_{n}$ for $n=7$ are known, European J. Combin. 11 (1990), no. 2, 115-117, URL: http://dx.doi.org/10.1016/S0195-6698(13)80064-9. doi:10.1016/S0195-6698(13)80064-9.

[29] C. Kuratowski, Sur le problème des courbes gauches en topologie, Fund. Math. 15 (1930), 271-283.

[30] P. D. Seymour, Matroids and multicommodity flows, European J. Combin. 2 (1981), no. 3, 257-290, URL: http://dx.doi.org/10.1016/S0195-6698(81)80033-9 doi:10.1016/S01956698(81)80033-9.

[31] M. E. Tylkin (=M. Deza), On Hamming geometry of unitary cubes, Soviet Physics. Dokl. 5 (1960), 940-943.

[32] K. Wagner, über eine Eigenschaft der ebene Komplexe, Math. Annal. 114 (1937), 570-590.

Michel Deza, École Normale Supérieure, Paris, Deceased

Mathieu Dutour Sikirić, Rudjer Bosković Institute, Bijenicka 54, 10000 Zagreb, Croatia, Fax: +385-1-468-0245

E-mail address: mathieu.dutour@gmail.com 